\title{
Perforation during esophageal submucosal dissection resulting from idiopathic partial muscular defect
}

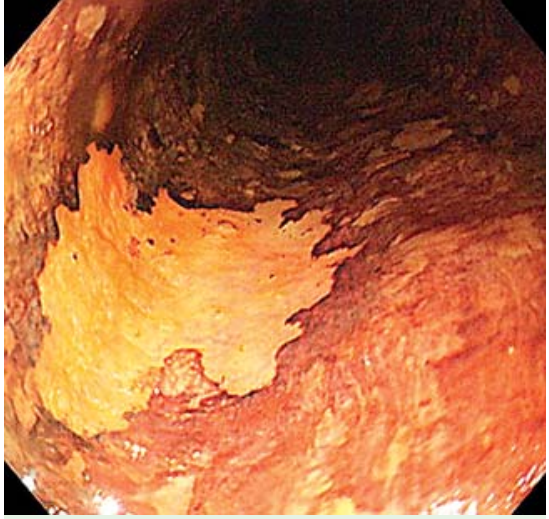

Fig. 1 Chromoendoscopy with iodine staining. A flat lesion, measuring $20 \mathrm{~mm}$ in diameter, was located on the left side of the middle thoracic esophagus.

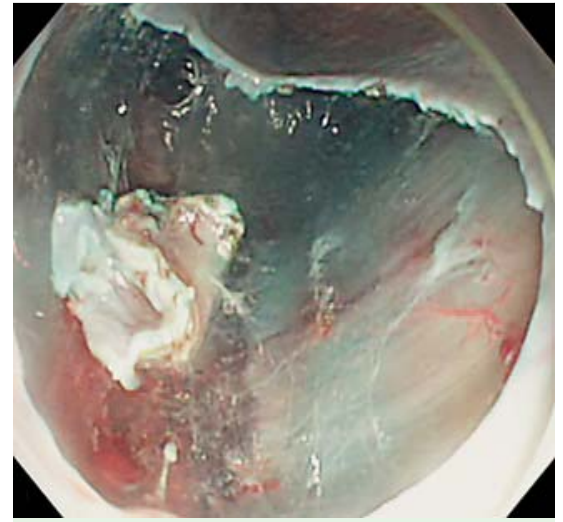

Fig. 2 Idiopathic partial defect of the muscularis propria layer was detected during submucosal dissection.

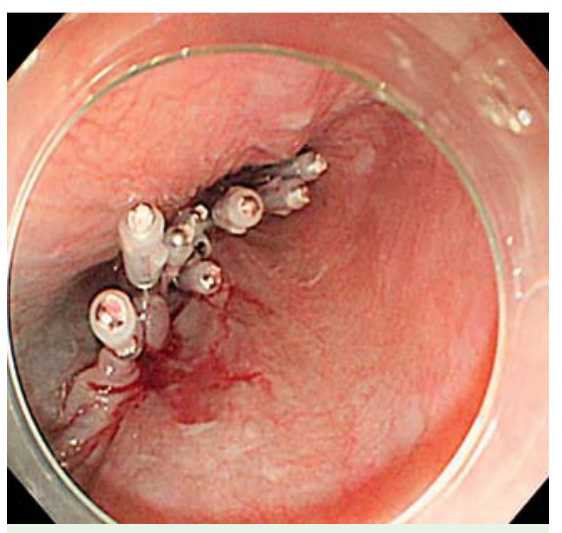

Fig. 4 The muscular defect was completely closed using clips.

Fig.3 Perforation occurred at the bottom of the muscular defect.

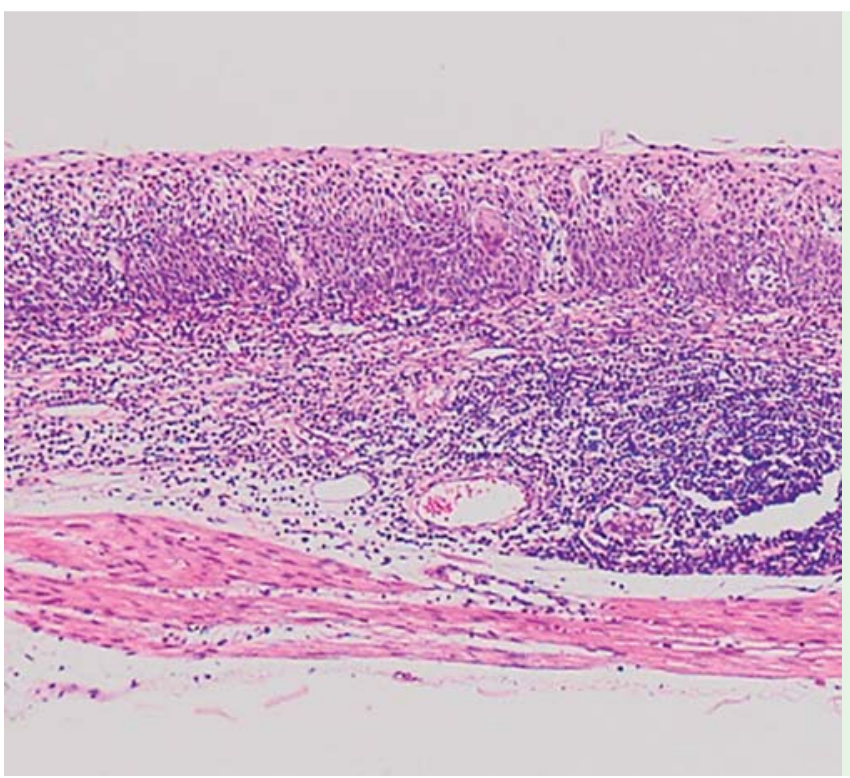

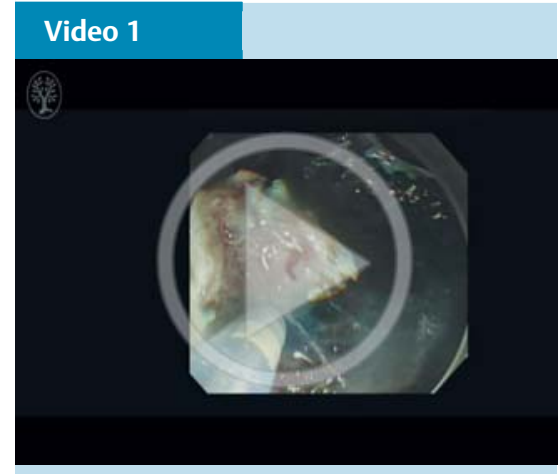

Endoscopic management of a perforation in a muscular defect during endoscopic submucosal dissection of a flat esophageal lesion.

Recently, endoscopic submucosal dissection (ESD) has been applied for the removal of superficial esophageal neoplasms [1]. Here, we report on the endoscopic management of a perforation during esophageal ESD, which resulted from an unexpected partial muscular defect.

An 80-year-old man underwent gastrointestinal endoscopy, and a lesion was detected on the left side of the middle thoracic esophagus ( $\bullet$ Fig. 1 ). The flat lesion, which measured $20 \mathrm{~mm}$ in diameter, was diagnosed as intraepithelial cancer.

ESD was performed by an experienced endoscopist using a Dual knife (KD650; Olympus Optical, Tokyo, Japan) and an IT knife nano (KD612; Olympus Optical) ( Video 1). Carbon dioxide was used for insufflation, following reports that it reduces postprocedural mediastinal emphysema [2]. After submucosal injection of sodium hyaluronate solution, circumferential incision was performed. During the submucosal dissection, an idiopathic partial defect of the muscularis propria layer was observed, although no mucosal depression that would indicate the presence of a diverticulum had been apparent $(\bullet$ Fig. 2). Despite the attentive procedure, perforation occurred at the bottom of the muscular defect ( $\bullet$ Fig.3). It was considered important to complete the procedure as quickly as possible, and therefore, submucosal dissection was abandoned and the lesion was removed using a snaring technique. The area of 
muscular defect was completely closed using clips ( $\bullet$ Fig.4).

Computed tomography scan after ESD revealed mild mediastinal emphysema, and the patient was treated with fasting and antibiotics, without the requirement for surgery. Pathological diagnosis indicated that squamous cell carcinoma was present within the epithelium, and that en bloc and curative resection had been achieved. The muscularis propria was not seen on the resected specimen ( $\nabla$ Fig.5), suggesting that the defect had not been caused by a technical error in the dissection depth. The causes and epidemiology of esophageal partial muscular defects have not been evaluated. To manage unexpected perforation during ESD procedures, the endoscopist needs to be proficient in closure techniques using clips.

Endoscopy_UCTN_Code_CPL_1AH_2AZ
Competing interests: None

\section{Tomoaki Tashima', Ken Ohata', Eiji Sakai ${ }^{1}$, Yohei Minato', Hideyuki Chiba², Kouichi Nonaka', Nobuyuki Matsuhashi ${ }^{1}$}

${ }^{1}$ Department of Gastroenterology, NTT Medical Center Tokyo, Tokyo, Japan

2 Department of Gastroenterology, Omori Red Cross Hospital, Tokyo, Japan

\section{References}

1 Probst A, Aust D, Markl B et al. Early esophageal cancer in Europe: endoscopic treatment by endoscopic submucosal dissection. Endoscopy 2015; 47: 113-121

2 Maeda $Y$, Hirasawa D, Fujita $N$ et al. A pilot study to assess mediastinal emphysema after esophageal endoscopic submucosal dissection with carbon dioxide insufflation. Endoscopy 2012; 44: 565-571
Bibliography

Dol http://dx.doi.org/ 10.1055/s-0042-102960

Endoscopy 2016; 48: E84-E85

(C) Georg Thieme Verlag KG

Stuttgart · New York

ISSN 0013-726X

Corresponding author

\section{Ken Ohata, MD}

Division of Gastroenterology

NTT Medical Center Tokyo

5-9-22 Higashi-gotanda Shinagawa-ku

Tokyo 141-8625

Japan

Fax: +81-3-34486541

ken.ohata1974@gmail.com 